\title{
The Role of Fascia Around the Patellar Tendon in Force Transmission: An Experimental Study on Sheep Stifle Joint
}

\author{
Hashem Yousefi ${ }^{* 1}$, Mahmoud Baniasadi ${ }^{2}$, Mostafa Rostami ${ }^{3}$ \\ Department of Biomedical Engineering, Amirkabir University of Technology, 424, Hafez Ave, Tehran, Iran. \\ ${ }^{* 1}$ ha.yousefi144@gmail.com; ${ }^{2}$ Mahmoud.Baniasadi@gmail.com; ${ }^{3}$ rostami@aut.ac.ir
}

\begin{abstract}
Myofascial force transmission was explained in different studies via extra, inter, and intramuscular pathways. In this work the role of fascia tissue around patellar tendon in force transmission was studied. In standard quasi static tension tests external loads were applied to stifle joint samples. Two types of experiments were performed, in which at the first one intact patellar tendon with tissue milieu was used as tissue sample, and at the second one its fascia tissue carefully removed. With applying a $5 \mathrm{~mm}$ axial displacement in both steps, results for force-displacement diagrams are collected. Compared results determined the linear distribution of transmitted force during the process. More than $\mathbf{1 5 - 2 0 \%}$ decrement in total force transmission was observed by carefully removing the fascia tissue around the patellar tendon.
\end{abstract}

Keywords- Fascia Tissue; Patellar Tendon; Force Transmission; Tension Test; Axial Displacement

\section{INTRODUCTION}

Investigating through the contribution of different muscles could be useful in understanding a better feature of joint motion [Maas, et al 2001]. This intent needs a principal source of force from muscle fibers, and this force needs to be transmitted through musculoskeletal system. As an example, different motions were studied for the individual force measurement in cat muscles tendons [Herzog et al, 1993] and later for in vivo human motion, different authors used ultrasonography for tendon elongation and movement measurements [Fukunaga, et al 1996 and Kubo, et al 1999]. Later works studied on the multi-finger motion of humans [Li et al 2000 and Zatsiorsky et al 2000]. With using of length as a general determinant of exerted force on muscles, Huijing and Baan detected effects of implementing extra loads on muscle belly which was transmitted as myofascial forces [Huijing \& Baan 2003]. This sentence carries the contribution of force transmission through fascia tissues.

In addition to transmitting from a muscle which is called myotendinous transmission of force, endomysial fascia can also transmit force through the muscle fibers [Rijkelijkhuizen, et al 2004]. Different pathways were recognized in myofasical force transmission; inter-, extra-, and intramuscular force transmission [Rijkelijkhuizen, et al 2004; Yucesoy, et al 2007]. Myofascial force transmission carries the concept of transmitting force through fascia or other connective tissues within a muscle. These pathways were determined to be involved in myofascial force transmission. Authors [Maas, et al 2001] studied extensor digitorum longus (EDL) in rat anterior crural force transmission with the variation in properties of extensor hallucis longus (EHL) and tibialis anterior (TA) muscles [Maas et al, 2001]. Some relevant studies showed that muscle fiber forces are transmitted to the other structures via (1): endo-, peri-, and epimysial network as the intramuscular connective tissues, (2): neurovascular tracts and extracellular matrix (ECM) of muscles to bone as the extra muscular connective tissues, and (3): at the belly-muscle interface and the direct connection of ECM in adjacent muscles as the intermuscular connective tissues [Purslow \& Trotter, 1994; Huijing et al., 1998; Huijing, 1999a; Huijing, 1999b; Huijing2000; Huijing \& Baan 2001; Yucesoy, et al 2003; Rijkelijkhuizen, et al 2004; Yucesoy, et al 2007]. Motion analysis of human body needs to evaluate the performance of each part in force transmission [Rijkelijkhuizen, et al 2004; Yucesoy, et al 2007]. Therefore, the main reason for the researchers to examine through the compartments in myofascial force transmission is to analyse and find out about the muscle skeleton system.

Rehabilitation process and the treatment period may affect with the contribution of fascia tissues in force transmission, which indeed shows its importance in joint movement. The motivation of this project was to investigate the effect of fascia tissue in force transmission around patellar tendon. Experiments in two stages were held on sheep stifle joint; at the first stage standard tension test implemented for intact patellar tendon with fascia tissue around, and at the second stage all fascia tissue removed from the joint. Main hypotheses of this paper: 1. Fascia tissues contribute in myofascial force transmission, 2. We would like to quantify its contribution on force transmission. It is expected that by carefully removing of the all fascia tissues in sheep stifle joint, it will affect on capacity of force transmission and it might be observed in force displacement diagram. Many numbers of tests were performed to investigate these hypotheses.

\section{MATERIALS AND METHODS}

The main steps for deriving the effects of fascia tissue in force transmission were disclosed with the proposed experiments on the test samples. Sheep stifle samples were used to study the difference between two mentioned stages. However, before 
performing the tests, it was needed to define and create the preliminary requirements of the experiments.

\section{A. Design of the Experiment}

Generally, results of the experimental procedure need a sufficient confidence, which is indeed followed by good simulation and professional design. In this case, general condition of the experiments, amount of load and type of external loadings, similarity in anatomy of the test samples, and design of appropriate fixture could be categorized in different design of experiments. Anatomically, human knee joint is very similar to stifle joints of animals such as dog, cat, sheep, and bovine [Yuehuei, H. et al, 2008]. Actually, the existing of fibula makes the Canine stifle joint the most similar one to human knee. Different features such as morality aspects, availability, weight, cost, illness sensitivity, easy handling and research history existence were considered as affecting features of selecting animal samples. Through the available samples sheep has the optimized level of consistency for our feature and do not have the rights' for human kind morality respection. Considering to the other facets such as availability, costs and easy handling, in this research the effects of fibula will be neglected.

\section{1) Sample Preparation:}

For each experiment four samples $(n=4)$ of young male sheep from the right stifle joint were considered to be examined for our studies. About 1 year old age and the weighting of $37.2 \pm 2.1 \mathrm{Kg}$ were used in our in-vitro studies.

Indeed, for these experiments we considered the homogenous types of tissues with respect to their normal physical conditions.

In experimental procedure, we need to remove most parts of connective tissues in knee joint unless patellar tendons and rounded fascias. This means for two different sets of the experiments we have fascia tissues removed from knee joint.

All the samples were kept between $4.5 \pm 0.5^{\circ} \mathrm{C}$ while dehydrated by spraying NSS of $(0.1 \mathrm{M})$. The room as the ambient temperature of $27.5 \pm 2.5^{\circ} \mathrm{C}$ and humidity of $75 \pm 2 \%$ was considered for experiment process.

\section{2) Zwick/Roell Device:}

Standard tension tests were employed with Zwick/Roell PC system (VERSION 5.1/17.01.92) testing machine. In these quasi-static tests, $5 \mathrm{~mm}$ displacement applied in axial direction. The resulted data of the standard tests in high frequency sampling rate were exploited in sketching of force-displacement graph. These graphs feature the axial displacement of patellar tendon and tissue milieu versus measured force up to $5 \mathrm{~mm}$.

\section{B. Preliminary Steps of the Experiment}

There are many limitations and requirements before performing of the experiments. Some of them could be mentioned such as: investigating the effects of dehydration and temperature in mechanical properties of soft tissues, designing of a tibia and patella fixture, bolt calibration in Pre-Tensioning Control System (PTCS), and elongation of connecting still wires. These steps might better to be considered before the performing.

\section{1) Dehydration and Temperature Effect:}

Between 65-70\% of tendon bring forth from water [Savio. L. et al, 2006], and dehydration will have undesirable effect on its mechanical properties. Side-effects of dehydration such as variation in mechanical properties of soft tissues had been confirmed in many previous studies. In case of test, and without normal saline spray, increment in module of elasticity and tensile strength of human patellar tendon is undeniable [Yuehuei, H. et al, 2008]. Therefore before the tests and during it, tendons and ligaments kept hydrated by spraying normal saline solutions (0.1 M Phosphate Buffered Saline). This will foreclose large variation in mechanical properties of patellar tendon and tissues milieu.

All of the test samples were kept at $4.5 \pm 0.5^{\circ} \mathrm{C}$, although some of the researcher discussed about the independency of the temperature in this kinds of tests [Yuehuei, H. et al, 2008]. All the samples were killed and obtained 5 hours before the tests and were performed in laboratory temperature of $27.5 \pm 2.5^{\circ} \mathrm{C}$.

\section{2) Tibia and Patella Fixture:}

In order to prevent torsion effects and provide axial rotation, it is needed to design a tibia fixture which could be employed at the desk of the testing machine. This part will be explained at the following steps. Considering Zwick/Roell testing machine desk, the motivation was to design a fixture which could be suitable for its special shape. Three fixing feet over the desk were employed for a better performance. Tibia could be fixed at the centre of the fixture by three bolts in $120^{\circ}$ angels. As it is obvious in Figure 1, displacement in longitudinal direction could be completely restricted with the generated notches on tibia and also could be filled by stone glue. The stone glue is a mixture of a main paste and a hardener paste. The mixture is completely like a liquid substance at first, which lead to its penetration in tibia notches. When the stone glue becomes solid tibia is completely limited in all directions. However, a thrust bearing has been used inside the fixture to eliminate torsion effects. 


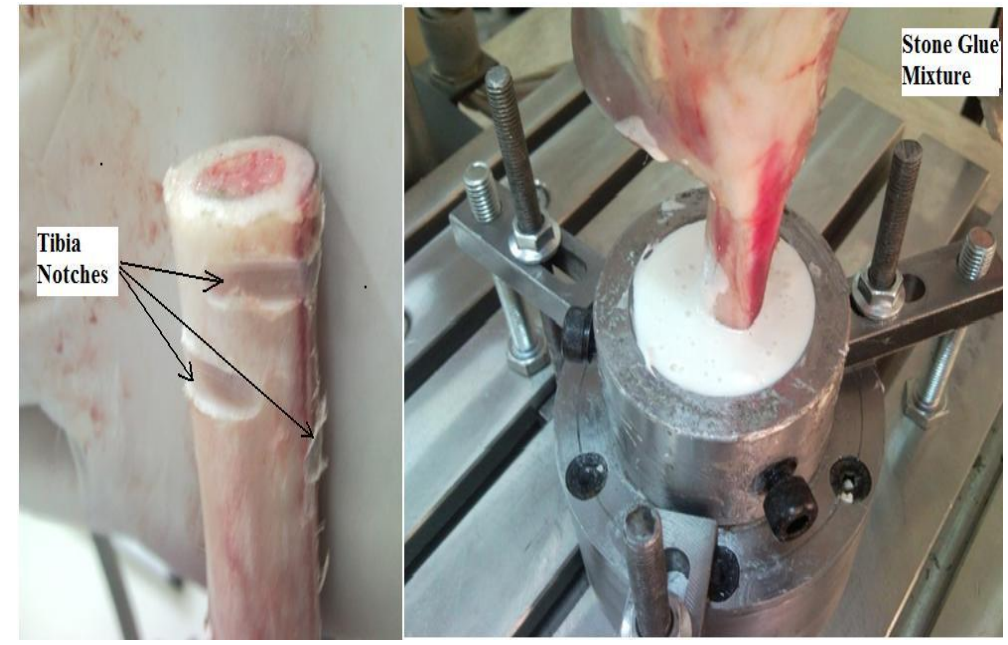

Figure 1- A) Better fixation with using some notches in tibia end-cut, - B) Tibia fixture with three holder bolts is filled by stone glue while notched tibia was kept at centre of fixture.

Gripping and pulling of the patella need to develop a novel mechanism. A gripper was attached to patella while a group of four steel wires (2-wires in each group) working as a connecting gripper to Pre-Tensioning Control System (PTCS is illustrated in Figure 2). In this condition it is needed to ensure the equally distribution of the applied tension force on all sides of the patella. This aim will be achieved with creating a notch on the gripper at an appropriate position for each wire. These notches will provide the solution of the wires' slipping problem with gripper contact. On the PTCS, each group of steel wires has been passed through a hole on a bolt and then fixed. Torque magnitude for fastening of each bolt is directly related to still wires' tension which is connected on it. The bolts on PTCS were utilized to create equal pre-tension in each steel wire and their positions were fixed by locking nuts.

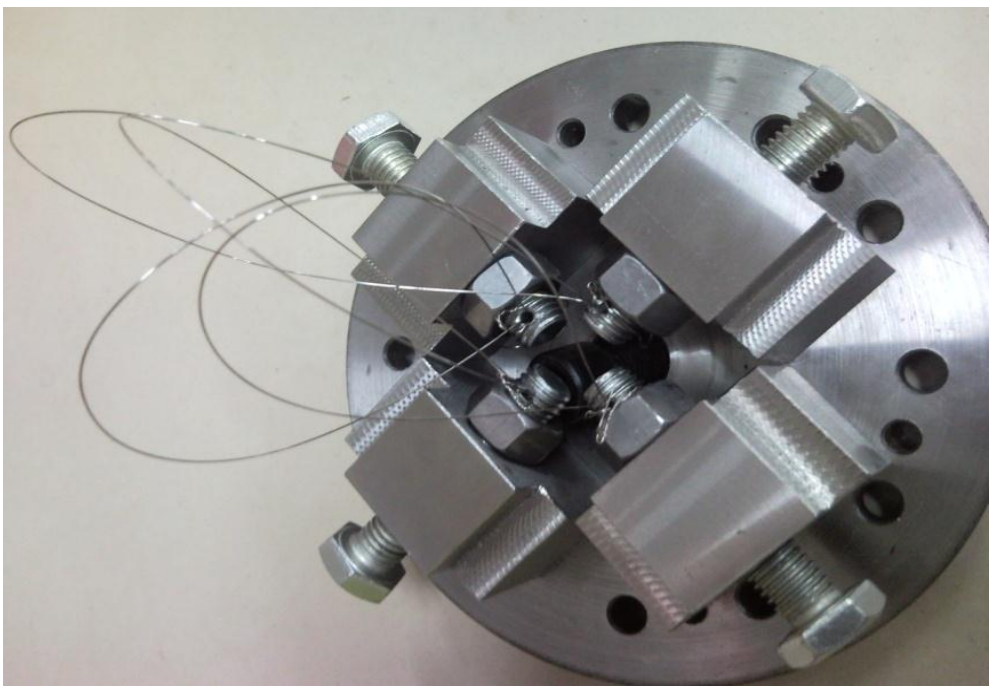

Figure 2- Pre-Tensioning Control System (PTCS)

In order to measure the amount of pre-tension in each group of steel wires without any effects on strain measurement, and torque magnitude for fastening the bolt was measured by a calibrated torque meter wrench. A simple calibration process for each bolt could be performed. This process also could be used in finding out the relation between fastening torque and pretensioning force. This is also could be mentioned that we performed a simple tension test for considering the elongation of steel wires. The elongation is due to tensioning force, and this test was performed prior to beginning of the main test.

\section{3) Bolt Calibration in PTCS:}

In order to find out the relationship between bolt fastening torque and tensioning force in PTCS, a simple experiment was performed; Sample wire was connected to a steel bar from one side and passed from hole of one bolt on PTCS and fastened to end of bolt at the other side. Steel bar sides were fixed to the testing machine bench. The tension force applied to steel wire by fastening the bolt. Torque was recorded from torquemeter respect to force recorded by tensile machine and repeated for 10 times for each torque, varies from 1.14 N.m to 2.48 N.m. Torque meter wrench was controlled by digital torque meter continuously, to ensure about applied torque magnitudes. 


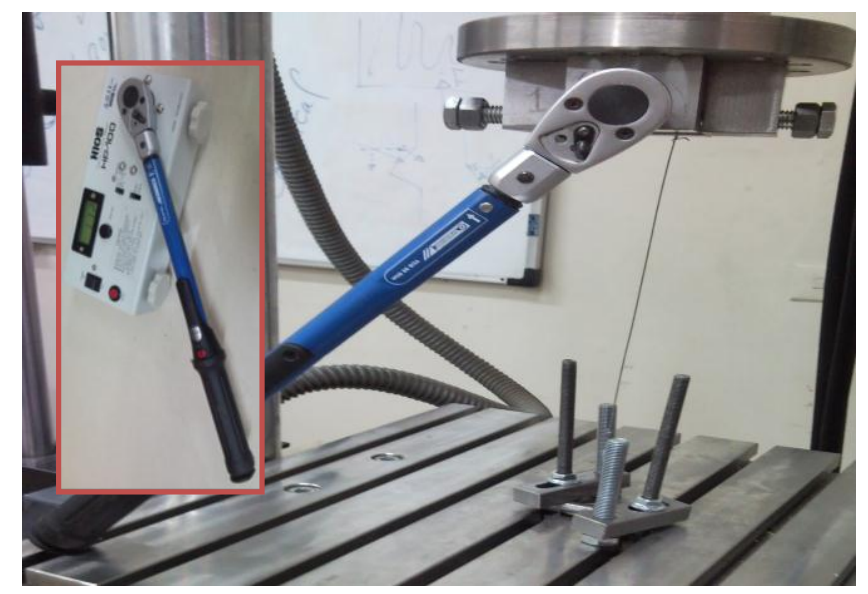

Figure 3 Calibrating the PTCS system using Torquemeter and Tensile testing machine to find out relation between fastening torque and wire tension force

All calibrating processes were performed for four bolts individually to consider probable manufacturing differences in PTCS bolts and nuts. Any possible physical differences may result in different fastening torques. The average recorded tensioning force was measured for each fastening torque. The resulted data set was used on plotting the fastening torques on these average tensioning forces. With the assumption of linear relation between force and torque, a unique formula was generated for each bolt. These formulas were considered to find out the appropriate fastening torques in applying equal pretensioning forces on each group of steel wires.

Although pre-tensioning forces must be applied as less as possible to prevent large strains in tendon tissue, it must be as much as possible to remove any losing state of steel wire groups to provide a uniform peripheral force on patella. Based on the results of the primary tests, $125 \mathrm{~N}$ force could be considered as the optimum magnitude for pre-tensioning. In this regard, the generated equations were used for applying and calculating the appropriate torques as well (Table I).

TABLE I: APPROPRIATE TORQUES OF EACH PTCS BOLTS IN CREATING EQUAL PRE-TENSIONS

\begin{tabular}{|c|c|c|c|c|}
\hline Bolt Number & 1 & 2 & 3 & 4 \\
\hline Pre-Tension Torque (N.m) & 1.58 & 1.87 & 1.54 & 1.83 \\
\hline
\end{tabular}

\section{4) Elongation of Steel Wires:}

Steel wires' elongation may better to be considered in preliminary steps of the main experiment. This effect could be studied with performing a simple test to find out the wire elongation. A $350 \mathrm{~mm}$ of steel wire was tested and the following diagram states its force-displacement diagram.

The maximum force that applied to patellar tendon was about $720 \mathrm{~N}$, which was applied to patella through 8 steel wires with the approximate length of $175 \mathrm{~mm}$. So the maximum elongation of steel wires in our test was equal to half of elongation of $350 \mathrm{~mm}$ wire when it is under $90 \mathrm{~N}$ tensioning forces. By tracking the force-displacement graphs (Figure 4), it's clear the maximum elongation of steel wires in our test would be $0.1 \mathrm{~mm}$. With respect to specimen strains, this is a negligible component of displacement to be considered in tests. Elongation is constant for both stages of the tests, before and after removing the fascia in tissue milieu of patellar tendon.

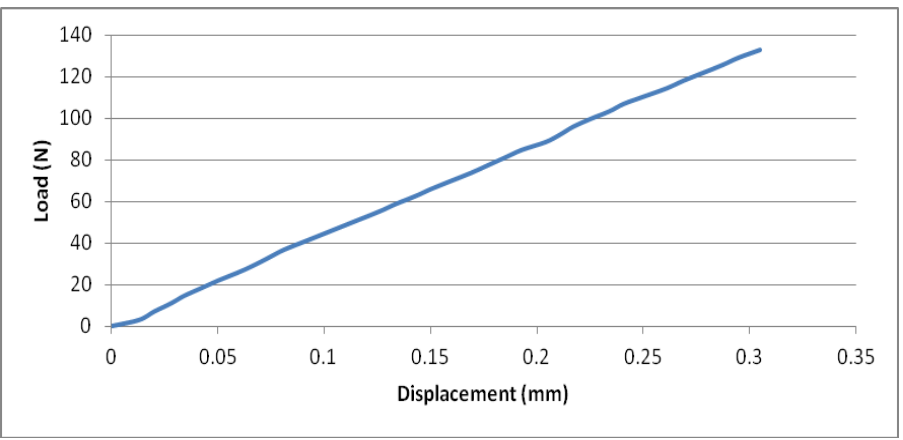

Figure 4 Force-displacement diagrams for a single $350 \mathrm{~mm}$ length steel wire in tension test

\section{Experimental Procedure}

In order to apply displacement to patellar tendon, patella fixture was used to attach patella bone to machine's clamp. During first step of dissection, all connections between patella and upper tissues (quad receipts and ...) had been removed 
carefully, leaving only patellar tendon and fascia in tissue milieu with intact patellar and tibial insertions. Therefore all of the measured forces were applied on the patellar tendon and fascia tissue. Eventually, the recorded data of the system provide force-displacement diagram for tension test at patellar tendon with fascia tissue.

In second step of dissection, fascia tissues around patellar tendon were carefully removed. The fascia tissues were cut up to hard tendon connection. The following picture (Figure 5) can give a better feature of the removed fascia.

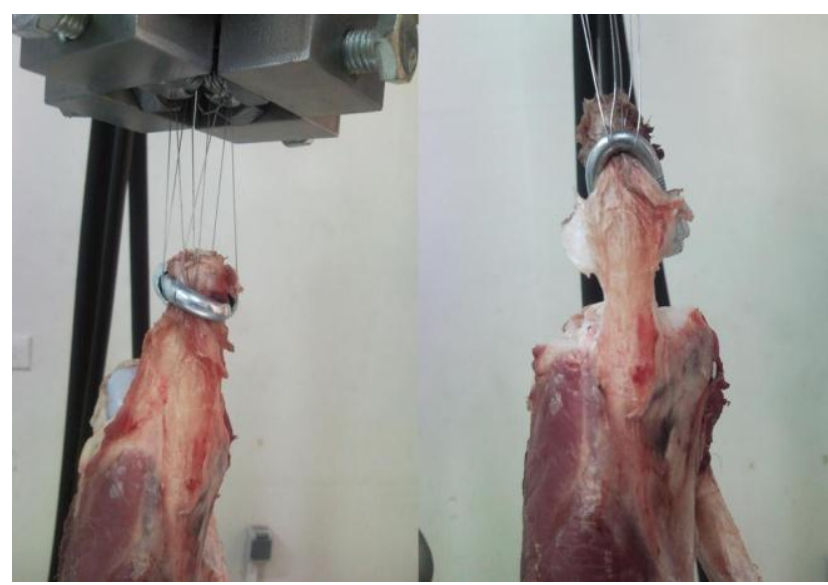

Figure 5 Removed fascia tissues from patellar tendon and tissue milieu.

In this step the patellar tendon is the only soft connecting tissue of the joint with intact tibial and patellar insertions. By exploiting the results of the first step and applying the same displacement to the new setup, force-displacement data set was generated for the second stage.

Simple tension tests were performed several times for each sample in Figure 6. Generally, tension tests were performed in quasi-static manner to investigate the main hypotheses. A $5 \mathrm{~mm}$ displacement with the rate of $1 \mathrm{~mm} / \mathrm{sec}$ was applied to tissue and was released to the origin condition immediately. These tests were performed 5 times for each sample before removing fascia tissue around patellar tendon and 5 times after removing fascia tissue around patellar tendon. To prevent hysteresis effect [Rittu V. et al, 2004] on soft tissue, a 5 minutes resting time (60 times of loading time) was considered between frequent tests.

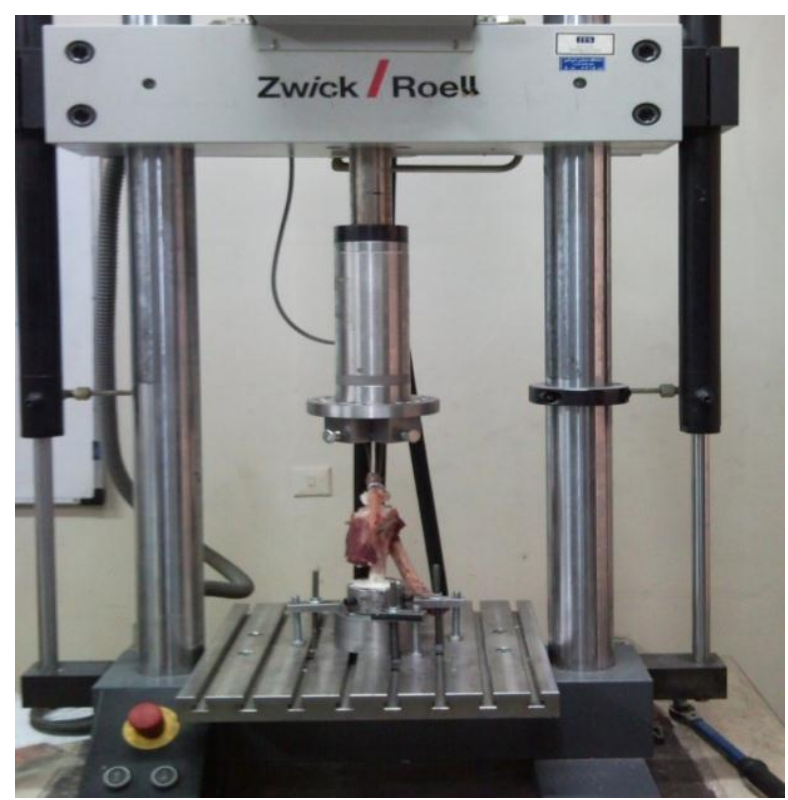

Figure 6 Simple Tension Tests were performed several times for each sample

\section{EXPERIMENTAL RESULTS}

Different sets of experimental data can be employed in achieving a comprehensive model for fascia tissue participation in force transmission. Experimental results should investigate and show the main hypotheses of this work. Therefore, the sample of the results of experiments in the first stage and the second stage were presented in Figure 7 and Figure 8, separately. In the given diagrams measured force was graphed versus the applied axial displacements. Summarized results were tabulated for a better judgment in Table 2. As it is obvious from the average results, for different axial displacements $15-20 \%$ decrement in 
force magnitude were measured.

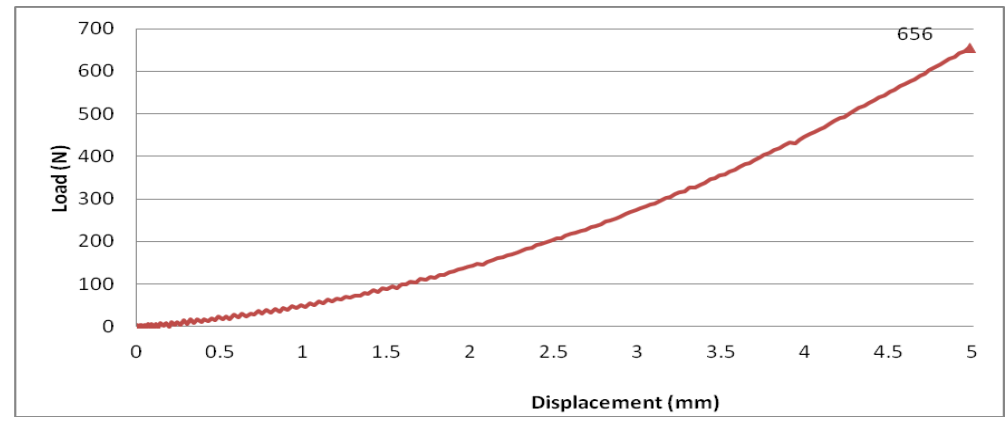

Fig. 7 Force-displacement diagram for sample \#2, before removing fascia tissue around patellar tendon

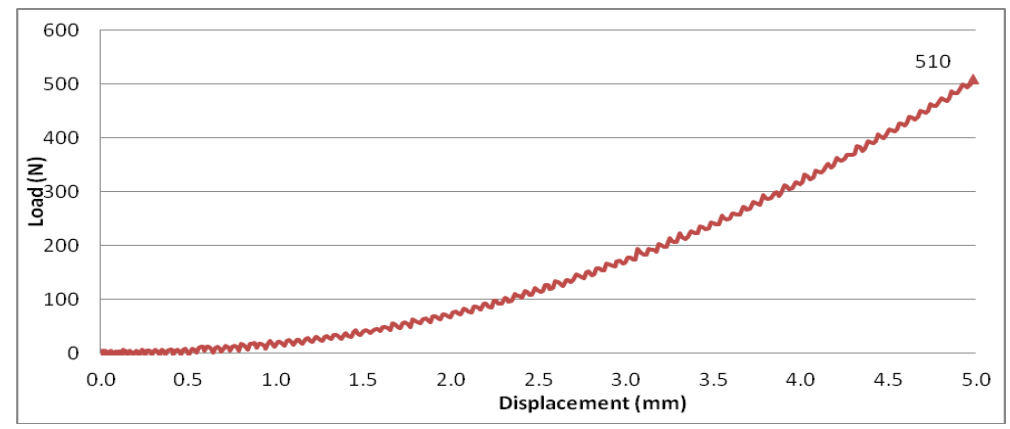

Fig. 8 Force-displacement diagram for sample \#2, after removing fascia in tendon tissue milieu

TABLE II GENERAL INFORMATION FOR THE RESULTS OF THE ALL SAMPLES; FORCE MAGNITUDE IN DIFFERENT DISPLACEMENTS UP TO 5MM

\begin{tabular}{cccccc}
\hline $\begin{array}{c}\text { All samples } \\
\text { (Displacement ) }\end{array}$ & $\mathbf{1 m m}$ & $\mathbf{2 m m}$ & $\mathbf{3 m m}$ & $\mathbf{4 m m}$ & $\mathbf{5 m m}$ \\
\hline $\begin{array}{c}\text { with fascia tissue } \\
\text { (force, } \mathbf{N} \text { ) }\end{array}$ & $48 \pm 4$ & $98 \pm 10$ & $149 \pm 21$ & $347 \pm 28$ & $627 \pm 61$ \\
$\begin{array}{c}\text { by removing fascia } \\
\text { tissue (force, } \mathbf{N} \text { ) }\end{array}$ & $42 \pm 6$ & $89 \pm 11$ & $137 \pm 16$ & $301 \pm 22$ & $509 \pm 29$ \\
\hline
\end{tabular}

Following diagrams can represent and confirm the mean hypotheses of the work, which was to quantify the effects of fascia tissue in force transmission with patellar tendon and its compartment in total force transmission of joint.

The following Table II carries the average information of the tension tests:

\section{IV.DISCUSSION}

In this study the authors were motivated to investigate two main hypotheses; they wanted to make sure that fascia tissue affect on force transmission in sheep stifle joint and also they wanted to quantify its compartment in force transmission. Regarded to the force-displacement diagram in Figure 9 which compares to different force measuring before and after removing fascia tissue around patellar tendon, difference in force for similar displacement is obvious. Results of the experiments features that the first half of diagrams are mostly "Toe Region" included, but the second half of diagrams show $15 \%-20 \%$ difference in force for similar displacement.

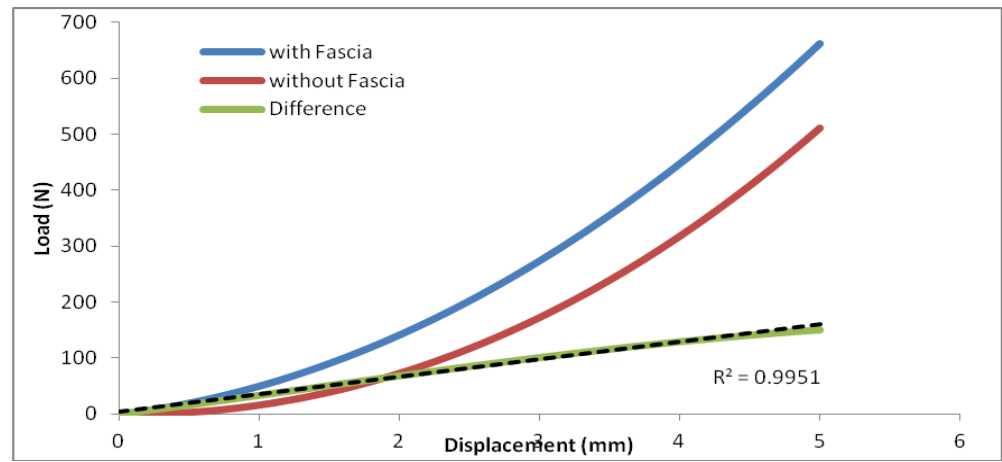

Figure 9 Displacement-Force Diagrams before and after removing fascia tissues and difference 
To discuss more about effect of fascias around patellar tendon, the force-displacement diagrams before and after removing fascia tissue around patellar tendon were drawn together, and 3rd diagram which is the result of subtraction of these two diagrams was added to them (Figure 9).

It is clear that the slope of first diagram is more than the slope of second diagram. It was expected because tension force passed through fascia tissue and patellar tendon parallels each other. This is also was shown in the expanded result of the Table II, with measured force on different step by step displacements. However, it is not so simple to refer the linear difference between these magnitudes of forces to simple viscoelastic models. The elastic module before removing fascia tissue is produced by summation of patellar tendon elastic module and fascia tissue elastic module. So the elastic module before removing fascia tissue is bigger than the state after removing fascia tissue.

The $3^{\text {rd }}$ diagram confirms this idea that its slope is positive. In addition, in this diagram, it is clear that the $3^{\text {rd }}$ diagram is linear with regression $\mathrm{R}^{2}=0.9951$. This means as displacement increases, the difference between first and second diagram increases.

\section{CONCLUSIONS}

To put it in a nut shell, these series of tests clearly and strongly confirm the main hypotheses. It is highly recommended to do a similar research in human patellar tendon. The result of this research would be useful for physiotherapists, surgeons and rehabilitation centres to improve their rehabilitation programs for tendon injuries patients.

Different studies (Zhang, 2005) provided novel methods for deriving mechanical properties of the biological tissue such as tendon. Applying Fast Fourier Transform (FFT) on stress-strain diagram measured in time can lead to some complex modules which contain the mechanical properties of the biological tissues. Under specified condition, this method can be employed without preliminary assumptions of tissue combination. This procedure can feature the future of this work.

It is needed to compare the results of the Fast Fourier Transform with available standard models. This comparison could have a confirmation to the accuracy of the FFT results.

\section{ACKNOWLEDGMENT}

We would like to thanks the Zwick/Roell operator Mr. Ehsan Sadeghian for his great performance on working with testing machine in Mechanical Behavior and Property Laboratory of Biomed Eng Dep, AUT, IR. Also Thanks to FaraTechnic Workshop Center stuffs for their help to prepare fixtures.

\section{REFERENCES}

[1] Franklin A. Young \& Yuehuei H. Animal Models in Orthopedic Research, Encyclopedia of Biomaterials and Biomechanical engineering, Volume 3, chapter 26, 2008 Informa Healthcare USA.

[2] Fukunaga, T., Ito, M., Ichinose, Y., Kuno, S., Kawakami, Y., Fukashiro, S. Tendinous movement of a human muscle during voluntary contractions determined by realtime ultrasonography. Journal of Applied Physiology 81, 1430-1433, 1996.

[3] Herzog, W., Leonard, T.R., Guimaraes, A.C. Forces in gastrocnemius, soleus, and plantaris tendons of the freely moving cat. Journal of Biomechanics 26, 945-953, 1993.

[4] Huijing, P.A. Muscle as a collagen fiber reinforced composite material: force transmission in muscle and whole limbs. Journal of Biomechanics 32, 329-345, 1999.

[5] Huijing, P.A. Muscular force transmission: a unified dual or multiple system? A review and some explorative experimental results. Archives of Physiology and Biochemistry 107, 292-311, 1999.

[6] Huijing, P.A. In vivo, force is transmitted from muscle also at other locations than the tendons: extramuscular myofascial force transmission. In: Proceedings of the Fifth Annual Congress of the European College of Sport Science, Jyv . askyl . a, Finland, 2000.

[7] Kubo, K., Kawakami, Y., Fukunaga, T. Influence of elastic properties of tendon structures on jump performance in humans. Journal of Applied Physiology 87, 2090-2096, 1999.

[8] Li, Z.M., Zatsiorsky, V.M., Latash, M.L. Contribution of the extrinsic and intrinsic hand muscles to the moments in finger joints. Clinical Biomechanics 15, 203-211, 2000.

[9] Maas. Huub, Guus C. Baan, Peter A, Huijing, Intermuscular interaction via myofascial force transmission: effects of tibialis anterior and extensor hallucis longus length on force- transmission from rat extensor digitorum longus muscle. Journal of Biomechanics 34, 927-940, 2001.

[10] Purslow, P.P., Trotter, J.A., The morphology and mechanical properties of endomysium in series-fibred muscles: variations with muscle length. Journal of Muscle Research and Cell Motility 15, 299-308, 1994.

[11] Rijkelijkhuizen J. M., Baan G. C., de Haan A., et al, Extramuscular myofascial force transmission for in situ rat medial gastrocnemius and plantaris muscles in progressive stages of dissection, The Journal of Experimental Biology 208, 129-140, 2004.

[12] Rittu V. Hingorani, Paolo P. Provenzano, Roderic S. Lakes, Anthony Escarcega, and Ray Vanderby Jr, Nonlinear Viscoelasticity in Rabbit Medial Collateral Ligament, Annals of Biomedical Engineering, Vol. 32, No. 2, February 2004, 306-312, 2003.

[13] Savio L. Y. Woo, Steven D. Abramowitch, Robert kilger, Rui Liang, Biomechanics of knee ligaments: injuriy, healing, and repair, Journal of Biomechanics 39, 1-20, 2006. 
[14] Yucesoy CA, Koopman HJFM, Baan GC, Grootenboer HJ, Huijing PA, Extramuscular myofascial force transmission: experiments and finite element modeling. Arch Physiol Biochem 111:377-388, 2003.

[15] Yucesoy. A, Koopman. B, Grootenboer, et al, Extramuscular myofascial force transmission alters substantially the acute effects of surgical aponeurotomy: assessment by finite element modeling, Biomechan Model Mechanobiol DOI 10.1007/s10237-007-0084-z.

[16] Yuehuei H. An, Richard J. Friedman, Animal Selections in Orthopaedic Research., Encyclopedia of Biomaterials and Biomechanical engineering, Volume 3, chapter 3, Informa Healthcare USA, 2008.

[17] Zatsiorsky, V.M., Li, Z.M., Latash, M.L. Enslaving effects in multi-finger force production. Experimental Brain Research 131, 187-195, 2000 .

Hashem Yousefi was born in Alamut, Qazvin, Iran, in April 1986. He received his B.Sc. degree from School of Mechanical Engineering, College of Engineering, University of Tehran, in 2004 and the M.Sc. degree in Biomechanics from the Biomedical Engineering Department at Amirkabir University of Technology (Tehran Polytechnic), in 2011. He is currently a member of Motion Analysis Laboratory, in the Department of Biomedical Engineering, Amirkabir University of Technology. His current research interests include robot-aided surgeries, image guided surgeries, rehabilitation robots, and soft tissue biomechanics. He has also worked on several projects in soft tissue finite element modeling, MEMS, dynamic systems, and control.

Mahmoud Baniasadi received a bachelor's degree in Mechanical Engineering from Sharif University of Technology in Iran in 2003. He received his M.Sc. Degree in Biomedical Engineering from the AmirKabir University of Technology in 2010. He has more than 8 years of professional working experiences as well he follows his research interests focus on biomechanics, Mechanical properties characterization and tissue engineering. Currently he is a member of Tissue Engineering Research team.

Mostafa Rostami received the M.S. and Ph.D. degree in robotics from the University of Poitiers, Poitiers, France, in 1995 and 1999. He is an associate professor at the Amirkabir University of Technology, Tehran, Iran. http://www.aut.ac.ir/rostami/. 